\title{
Erratum to: Attentional control in the attentional blink is modulated by odor
}

\author{
Lorenza S. Colzato $^{1,2} \cdot$ Roberta Sellaro $^{1} \cdot$ Claudia Rossi Paccani $^{1} \cdot$ Bernhard Hommel $^{1}$
}

Published online: 28 May 2015

(C) The Psychonomic Society, Inc. 2015

\section{Erratum to: Atten Percept Psychophys \\ DOI 10.3758/s13414-014-0733-0}

Please find below a list of corrections made to this article.

1) Two sentences in the article's General method section should be eliminated because they have been incorrectly reported. These sentences appear under the subheading Statistical analysis on page 1512 and should read:

"For both Experiments 1 and 2, an a priori power analysis was performed to estimate the approximate number of participants required for detecting medium effect sizes $(\approx .40)$, given the traditional .05 criterion of statistical significance. The calculation was made using G*Power 3.1.7 program (Faul, Erdfelder, Lang, \& Buchner, 2007).”

The online version of the original article can be found at http://dx.doi.org/ 10.3758/s13414-014-0733-0.

Lorenza S. Colzato

colzato@fsw.leidenuniv.nl

1 Institute for Psychological Research \& Leiden Institute for Brain and Cognition, Leiden University, Leiden, The Netherlands

2 Cognitive Psychology Unit, Leiden University, Wassenaarseweg 52, 2333 AK Leiden, The Netherlands
2) A mistake occurred while reporting the results of the mood analyses of Experiment 1 ( page 1513, last paragraph of the Results section): the data for the control group were not included in the analyses.

The amended formulation appears below.

"The mood ANOVA showed that participants tended to experience less arousal at the second than at the first measurement $(0.6$ vs. 0.07$), F(1,61)=7.42, p=.008, \eta_{p}^{2}=.11$, whereas pleasure levels were constant across the two measurements ( 0.9 vs. 0.6 ), $\mathrm{F}(1,61)=2.06, \mathrm{p}=.16, \eta_{\mathrm{p}}^{2}=.03$. Importantly, for both arousal $(0.4$ vs. $0.0,0.8$ vs. 0.4 , and 0.5 vs. -0.3 for lavender, peppermint, and control, respectively) and pleasure ( 0.9 vs. $0.5,0.6$ vs. 0.7 , and 1.1 vs. 0.7 for lavender, peppermint, and control, respectively) analyses, neither the main effect of group nor the interaction between group and time reached significance levels, $\mathrm{F}_{\mathrm{s}}<1.1, \mathrm{p}_{\mathrm{s}} \geq .34$." 